\title{
A UNIVERSAL CALIBRATION FOR GEL PERMEATION CHROMATOGRAPHY
}

Gel permeation chromatography is one of the most powerful techniques for characterizing the polydispersity of polymeric materials (1). A versatile commercial apparatus (2) has been used successfully in numerous laboratories on various problems of molecular weight distributions. But one of the difficulties still unsolved is the problem of calibration, i.e., the relation between elution volume and molecular weight.

Some authors (2) have assumed that retention time depends on the contour length of the molecular chain. Others think that it is more reasonable to use the radius of gyration or some average volume of the polymer molecule as the calibration parameter.

In a recent paper (3) we have reported GPC retention times of a series of polystyrenes exhibiting different molecular structures: linear, star-shaped, and comb-like. All were of known molecular weight and of low polydispersity. Obviously the conventional calibration method, where the logarithm of molecular weight is plotted against elution volume, does not yield a universal curve for all the samples. Retention times for branched samples were always larger than those for the linear homologs of the same molecular weight. We therefore tried using the hydrodynamic volume as the calibration parameter.

According to the Einstein viscosity law one can write

$$
[\eta]=K(V / M)
$$

where $[\eta]$ is the limiting viscosity index, $\mathrm{V}$ the hydrodynamic volume of the particles, $M$ their molecular weight, and $K$ a constant. This equation shows that the product $[\eta] \mathrm{M}$ is a direct measure of the hydrodynamic volume of the particles and suggests the use of $\log [\eta] \mathrm{M}$, instead of $\log$ $\mathrm{M}$, in the calibration of the chromatograms. With this type of plot, all our experimental points fall on the same curve. This assertion is supported by the following considerations. It is well known that for branched polymers one can write

$$
[\eta] \mathrm{M}=\phi \mathrm{R}_{\mathrm{L}}^{3} \mathrm{~g}^{\mathrm{x}}
$$

or

$$
[\eta] M=\phi^{\prime} R^{3} g^{x}-3 / 2
$$

where $R$ is the actual radius of gyration, $g$ is the parameter introduced 
by Zimm and Stockmayer (4), $\phi$ is a universal constant, and $\mathrm{x}$ is an exponent ranging from $1 / 2$ to $3 / 2$ depending upon the theory used.

If this is true, it means that it is the hydrodynamic volume-obtained from viscometric data - which determines retention in the chromatographic columns. But if the hydrodynamic volume is the parameter responsible for GPC retention, the above calibration should be valid for any polymer, regardless of its chemical nature as well as of its morphological structure: it should be universal, and thus be characteristic for any given set of columns and elution solvent at a given temperature. We have measured GPC elution volumes of a number of polymer samples chosen for their small polydispersity. The polymers, obtained in most cases through anionic reaction mechanisms, were: polystyrene (PS), poly(methyl methacrylate) (PMM), polybutadiene, and poly(vinyl chloride) samples which can be considered as having linear molecular structure; block and graft copolymers of styrene and methyl methacrylate; star-shaped polystyrenes and poly(methyl methacrylates); "heterograft" copolymers composed of a three-block sequence PMM-PS-PMM, with PS grafts on the PMM blocks.

Molecular weights $M$ were determined by light scattering (Sofica apparatus) in suitable solvents; control of polydispersity rendered it necessary, sometimes, to get number-average values through osmometry (Mechrolab equipment).

Intrinsic viscosities were measured, for all samples, on tetrahydrofuran solutions in a capillary viscometer at $25^{\circ} \mathrm{C}$.

The gel permeation chromatography experiments were carried out on a Waters machine equipped with 4 columns $\left(10^{6}, 10^{5}, 10^{4}, 9 \times 10^{2} \mathrm{~A}\right.$.), at room temperature. The solvent was tetrahydrofuran. Injection time was $2 \mathrm{~min}$. and pumping rate was always $1 \mathrm{ml} . / \mathrm{min}$. All the chromatograms were quite sharp and almost symmetric. The maximum of the peak was taken as the elution volume for each of the tested polymers and the $\log$ of the product $[\eta] \mathrm{M}$ was plotted as a function of this elution volume, which was measured in 5 -ml. increments (counts).

The results are collected in Table I, and a plot is shown in Figure 1.

It can be seen that all of the experimental points lie on a single curve. This confirms our hypothesis according to which the viscometric hydrodynamic volume, characterized by $[\eta] M$, determines retention in the chromatographic column. It is interesting to note that this universal calibration curve takes into account interactions of all types-those between polymer and solvent, and in the case of copolymers the heterocontact interactions-which are included in $[\eta]$. This explains why molecular weight calibration curves, i.e., $\log M$ vs. elution volume, established for each homologous series exhibit different slopes (Fig. 2): in a given solvent the viscosity laws for different polymers have different exponents. 


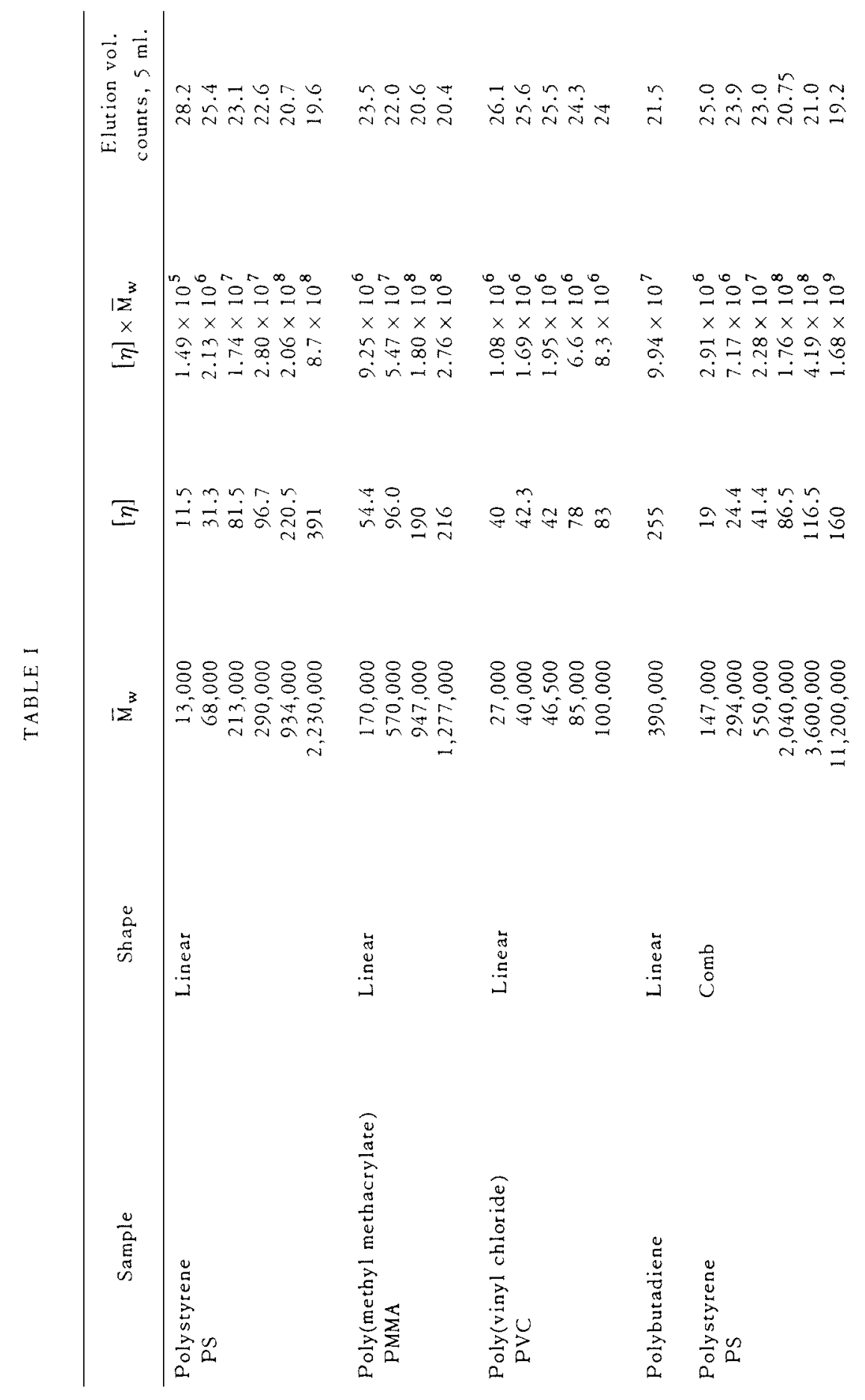




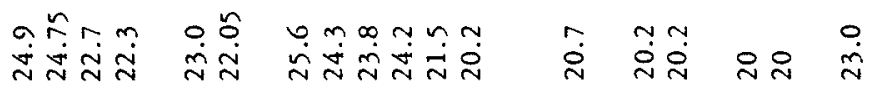

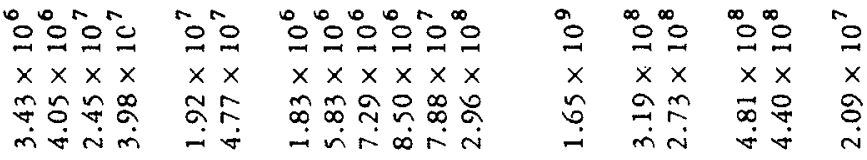

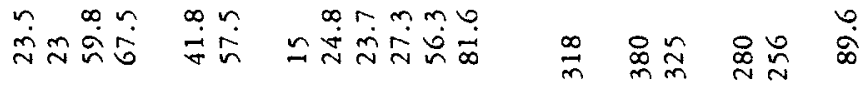

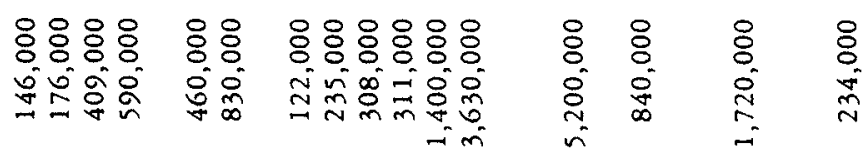

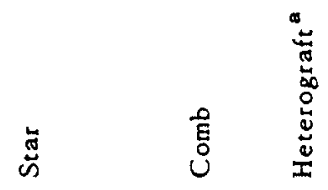

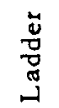

$\frac{\text { a }}{\text { ch }}$
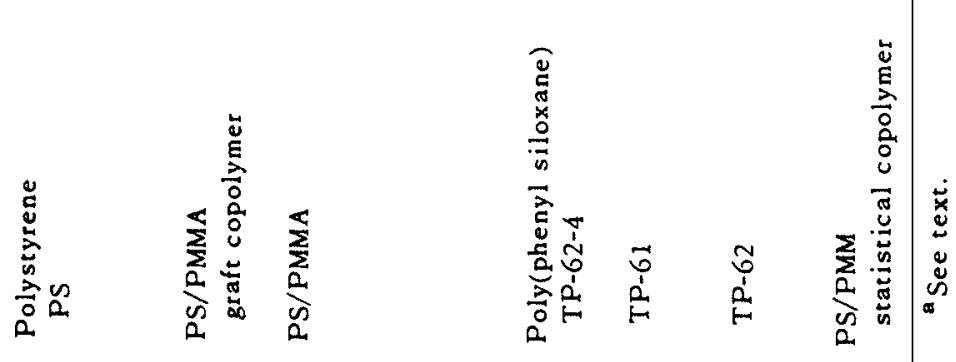


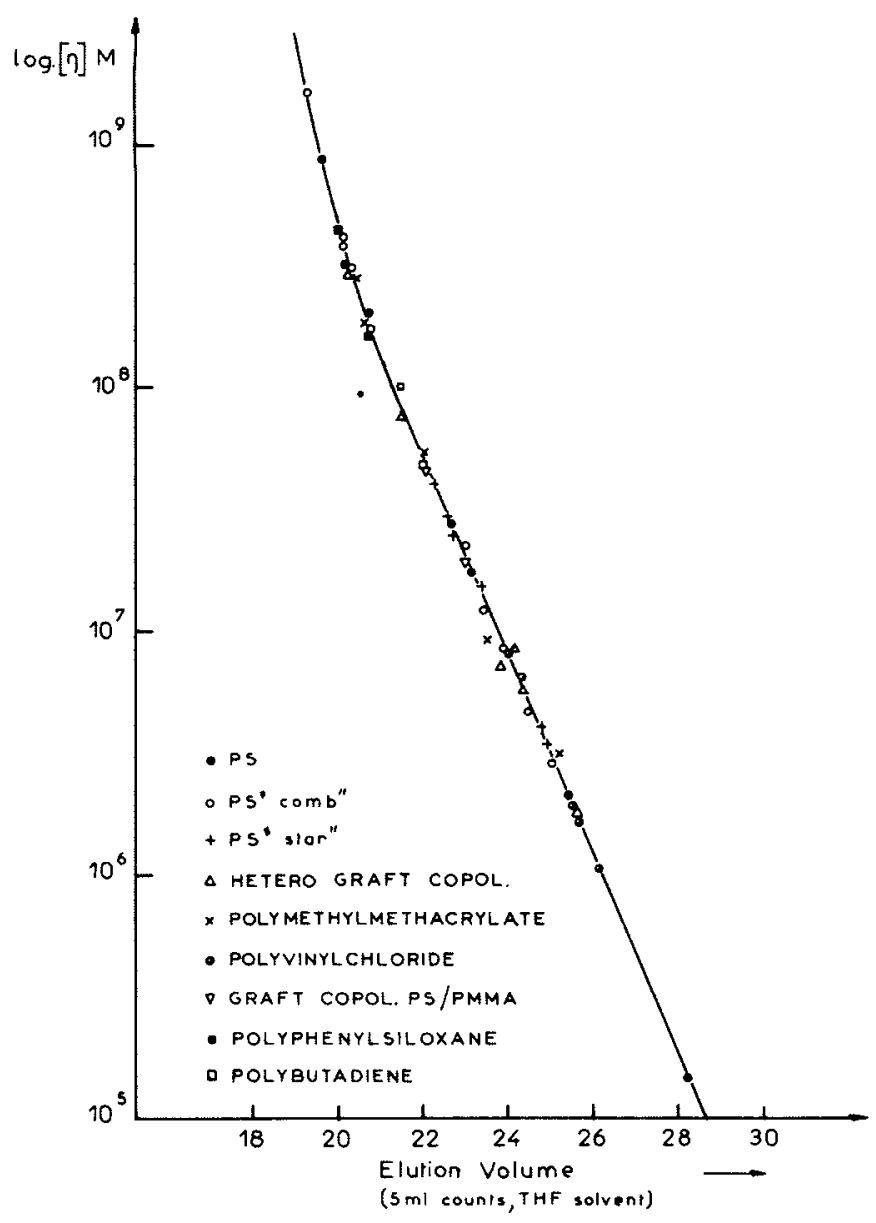

Figure 1.

Recently it has been shown (4) that the $\log [\eta] M$ plot also furnishes a good fit for polystyrene and for poly(L-benzyl glutamate) in dimethylformamide. It is well known that the shape of these molecules is quite different: the first is a coil, the other a rigid rodlike molecule. Therefore, this result suggests that our calibration is independent of the shape of the molecules and is valid for elongated particles as well.

Recently, Meyerhoff (5) published his own results in this field, and according to him the plots of $\log [\eta]^{1 / 3} \mathrm{M}^{1 / 2}$ vs. elution volume should yield parallels for different polymeric series. Although the theoretical basis of this plot is not very convincing, we plotted our own experimental results according to Meyerhoff and obtained for each type of polymer a different straight line, but all the lines were parallel. Thus 


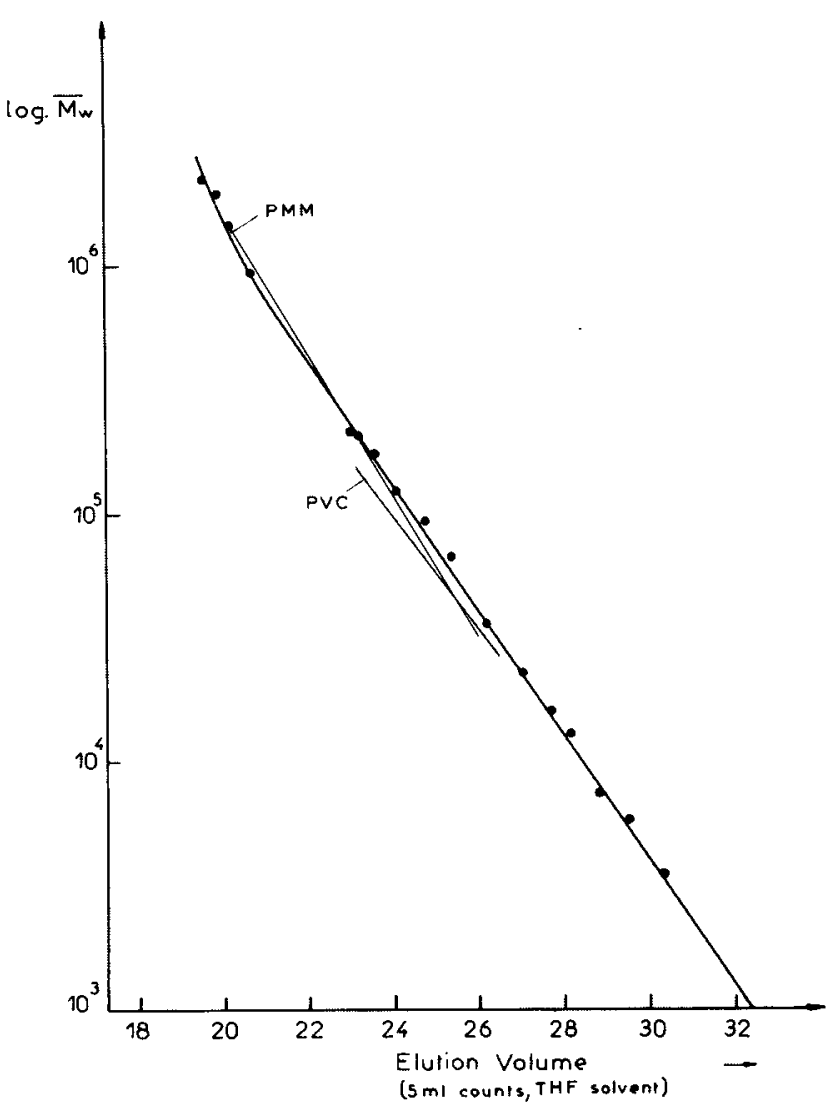

Fig. 2. GPC calibration: polystyrene/THF.

it is demonstrated that the calibration proposed by Meyerhoff cannot be considered universal, since different polymeric series yield different curves for the same set of columns, the same solvent, and the same temperature.

The question now arises if it would not be possible to obtain the same kind of results using the radius of gyration. From our data on graft polymers this seems not to be the case. The value of $\mathrm{x}$ in eq. (3) is often assumed to be $1 / 2$ (Zimm and Kilb) (7). Since in our samples $\mathrm{g}$ values range from 1 to 0.2 , there is for the most highly branched polymers a factor of five between hydrodynamic and geometrical volumes, which makes it impossible to have a good fit in both representations.

It is somewhat surprising to obtain such a good fit with the volume obtained viscometrically, and one could ask why another hydrodynamic volume, such as that obtained from translational brownian motion, could not be used. If one looks at the molecules moving in front of the pores 
in a velocity gradient, just as in a viscosity experiment, one could assume that it is the volume perturbing the flow that also governs the entry of molecules into the pores. But this is merely a hypothesis.

Even if this explanation is not valid, this new method of calibration seems promising. GPC results can be considered as a combination of molecular weight and viscosity. Owing to the ease of both GPC and viscosity experiments, this method can be used for molecular weight determinations on unknown polymers, and it should be especially useful and efficient for polymers soluble only at elevated temperatures.

We thank Mrs. Decker, Messrs. Curchod, Guyot, Gallot, and Zilliox for some of the samples we have used.

\section{References}

(1) J. C. Moore, J. Polymer Sci. A, 2, 835 (1964).

(2) L. E. Maley, in Analysis and Fractionation of Polymers (J. Polymer Sci. C, 8), J. Mitchell, Jr. and F.W. Billmeyer, Jr., Eds., Interscience, New York, 1965, P. 253.

(3) H. Benoit, Z. Grubisic, P. Rempp, D. Decker, and J. G. Zilliox, J. Chim. Phys., 63, 1507 (1966).

(4) B. H. Zimm and W. H. Stockmayer, J. Chem. Phys., 17, 1301 (1949).

(5) Z. Grubisic, L. Reibel, and G. Spach, Compt. Rend., in press.

(6) G. Meyerhoff, Makromol. Chem., 89, 282 (1965);

G. Meyerhoff, Ber. Bunsenges. Physik., 69, 866 (1965).

(7) B. Zimm and R. W. Kilb, J. Polymer Sci., 37, 19 (1959).

Z. Grubisic

P. RempP

H. Benoit

Centre de Recherches sur les Macromolécules

Strasbourg, France

Received March 27, 1967

Revised May 22, 1967 